\title{
Impact of Mitochondrial Targeting Antibiotics on Mitochondrial Function and Proliferation of Cancer Cells
}

\author{
Edward J. Cochrane ${ }^{\ddagger}$, James Hulit ${ }^{\dagger}$, Franz P. Lagasse ${ }^{\ddagger}$, Tanguy Lechertier ${ }^{\dagger}$, Brett Stevenson ${ }^{\ddagger}$, Corina \\ Tudor $^{\dagger}$, Diana Trebicka ${ }^{\dagger}$, Tim Sparey ${ }^{\dagger}$, Andrew J. Ratcliffe ${ }^{\dagger *}$ \\ ${ }^{\dagger}$ Novintum Biosciences Ltd, London Bioscience Innovation Centre, 2 Royal College Street, London, NW1 0NH, United \\ Kingdom \\ * Department of Chemistry, Sygnature Discovery, BioCity, Pennyfoot Street, Nottingham NG1 1GR, United Kingdom
}

\section{Synthetic Procedures}

Unless otherwise noted, all reagents were obtained from commercial suppliers and used without further purification. Product yields are not optimised. Reactions were monitored on TLC Xtra SIL G Silica Layers on Alugram ${ }^{\mathrm{TM}}$ Aluminum Sheets UV254 or by HPLC analysis with Waters SQ mass detector. Flash chromatography was performed on Buchi Pure C-810 (pre packed columns 60-120 mesh silica). NMR spectra were obtained on a $400 \mathrm{MHz}$ Bruker AV III or $500 \mathrm{MHz}$ AV III HD using dimethylsulfoxide- $d_{6}$ (DMSO- $d_{6}$ ) and methanol- $d_{4}$ $\left(\mathrm{CD}_{3} \mathrm{OD}\right)$ as received. Abbreviations used in describing peak signals are: br $=$ broad signal, $\mathrm{s}$ $=$ singlet, $\mathrm{d}=$ doublet, $\mathrm{dd}=$ doublet of doublets, $\mathrm{tt}=$ triplet of triplets, sep $=$ septet, $\mathrm{m}=$ multiplet. High resolution mass spectroscopy (HRMS) was carried out using a Waters quadrupole time of flight mass spec (Xevo G2-XS Q-Tof) and cortecs UPLC C18, $1.6 \mu \mathrm{m}, 2.1$ x $30 \mathrm{~mm}$ column eluting with a gradient of $\mathrm{MeCN}$ in $0.1 \%$ formic acid. Column equilibration from 0.0 to $0.1 \mathrm{~min}$ and then a gradient from 5-95\%. Analytical HPLC analyses were performed on a Waters SQ mass detector instrument using one of the following reverse phase methods:

Method A Column: Waters Acquity UPLC BEH C18, $1.7 \mu \mathrm{m}, 2.1$ x 30 mm; Gradient Eluent: $5-95 \% \mathrm{MeCN} / \mathrm{H}_{2} \mathrm{O}$ containing $10 \mathrm{mM}\left(\mathrm{NH}_{4}\right)_{2} \mathrm{CO}_{3}$; Time: 0-3 min

Method B Column: Waters Acquity UPLC CSH C18, $1.7 \mu \mathrm{m}, 2.1$ x $30 \mathrm{~mm}$; Gradient Eluent: 5-95\% MeCN/ $\mathrm{H}_{2} \mathrm{O}$ containing 0.1\% $\mathrm{HCOOH}$; Time: 0-10 min

All target molecules 1-10 and $\mathbf{1 8}$ were found to be $>95 \%$ by LC/MS.

\{12-[(2R,3S,4R,5R,8R,10R,11R,12S,13S,14R)-11- $\{[(2 S, 3 R, 4 S, 6 R)-4-(d i m e t h y l a m i n o)-3-$ hydroxy-6-methyloxan-2-yl]oxy\}-2-ethyl-3,4,10-trihydroxy-13-\{[(2R,4R,5S,6S)-5hydroxy-4-methoxy-4,6-dimethyloxan-2-yl]oxy\}-3,5,8,10,12,14-hexamethyl-15-oxo-1oxa-6-azacyclopentadecan-6-yl]-12-oxododecyl \}triphenylphosphonium hexafluorophosphate(V) (1) 


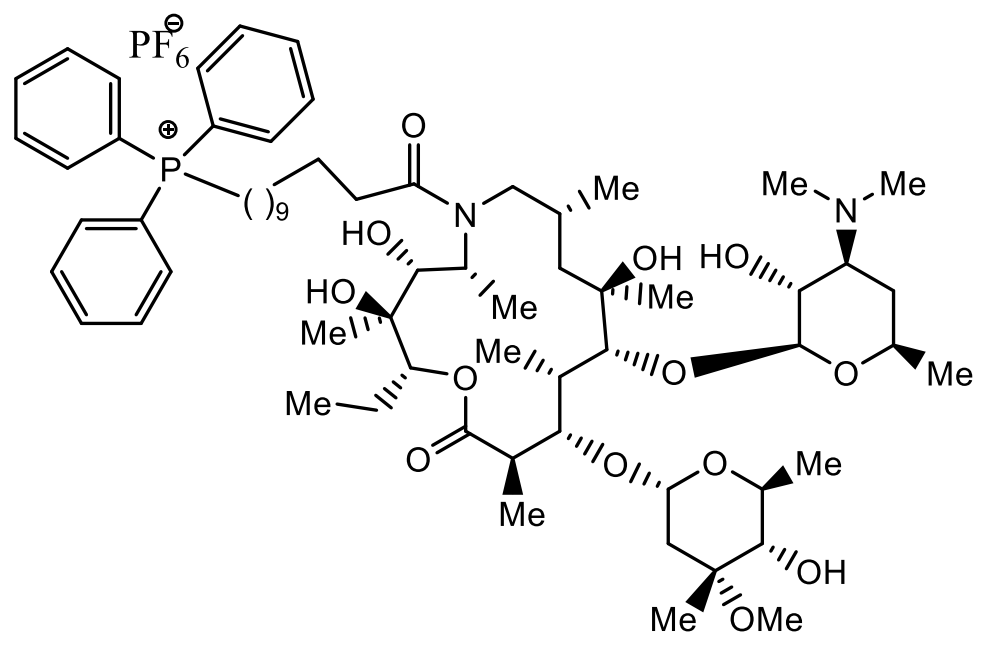

A solution of (11-carboxyundecyl)triphenylphosphonium bromide (0.61 g, $1.13 \mathrm{mmol}), 1$ [bis(dimethylamino)methylene]-1H-1,2,3-triazolo[4,5-b]pyridinium 3-oxid hexafluorophosphate (HATU) (0.43 g, $1.13 \mathrm{mmol})$ and N,N'-diisopropylethylamine (DIEA) $(0.54 \mathrm{~mL}, 3.1 \mathrm{mmol})$ in DCM $(7 \mathrm{~mL})$ was stirred at room temperature. After $30 \mathrm{~min} 9 \mathrm{a}-\mathrm{N}-$ desmethyl-azithromycin $(0.75 \mathrm{~g}, 1.02 \mathrm{mmol})$ was added and the reaction mixture further stirred at room temperature. After $16 \mathrm{~h}$ the reaction mixture was treated with saturated aqueous $\mathrm{NaHCO}_{3}(5 \mathrm{~mL})$ and DCM $(5 \mathrm{~mL})$, and passed through a phase separator, washing with DCM $(2 \times 5 \mathrm{~mL})$. The collected solvent was removed under reduced pressure. The resulting residue was taken up in $7 \mathrm{M} \mathrm{NH}_{3} / \mathrm{MeOH}(5 \mathrm{~mL})$ and stirred at room temperature. After $1 \mathrm{~h}$ the solvent was removed under reduced pressure and the residue partitioned between EtOAc $(15 \mathrm{~mL})$ and saturated aqueous $\mathrm{NaHCO}_{3}(15 \mathrm{~mL})$. The aqueous layer was separated and extracted with EtOAc $(15 \mathrm{~mL})$. The combined organic extracts were washed sequentially with saturated aqueous $\mathrm{NaHCO}_{3}(15 \mathrm{~mL})$, saturated aqueous $\mathrm{NH}_{4} \mathrm{Cl}(2 \times 15 \mathrm{~mL})$, brine $(30 \mathrm{~mL})$, dried over $\mathrm{MgSO}_{4}$, filtered and the solvent removed under reduced pressure. The resulting residue was purified by silica column chromatography eluting with $\left.0-5 \%\left(0.7 \mathrm{M} \mathrm{NH}_{3} / \mathrm{MeOH}\right) / \mathrm{DCM}\right)$ to give the title compound 1 (49 mg, $4 \%$ yield) as a white solid; ${ }^{1} \mathrm{H}$ NMR (400 MHz, DMSO$\left.d_{6}\right): \delta$ (delta) ppm 7.94-7.86 (m, 3H), 7.85-7.70 (m, 12H), $5.06(\mathrm{~d}, J=10.1 \mathrm{~Hz}, 1 \mathrm{H}), 4.76(\mathrm{~d}, J$ $=4.8 \mathrm{~Hz}, 1 \mathrm{H}), 4.42(\mathrm{~d}, J=7.1 \mathrm{~Hz}, 1 \mathrm{H}), 4.29(\mathrm{~d}, J=7.5 \mathrm{~Hz}, 1 \mathrm{H}), 4.22(\mathrm{~s}, 1 \mathrm{H}), 4.04(\mathrm{~d}, J=7.9$ $\mathrm{Hz}, 1 \mathrm{H}), 3.93(\mathrm{~d}, J=8.6 \mathrm{~Hz}, 1 \mathrm{H}), 3.70-3.61(\mathrm{~m}, 2 \mathrm{H}), 3.60-3.49(\mathrm{~m}, 1 \mathrm{H}), 3.42-3.35(\mathrm{~m}, 1 \mathrm{H})$, 3.28-3.22 (m, 1H), 3.19 (s, 3H), 3.09-2.95 (m, 3H), 2.94-2.88 (m, 2H), 2.69-2.63 (m, 1H), 2.28$2.09(\mathrm{~m}, 8 \mathrm{H}), 1.82-1.72(\mathrm{~m}, 1 \mathrm{H}), 1.71-1.36(\mathrm{~m}, 12 \mathrm{H}), 1.35-0.95(\mathrm{~m}, 40 \mathrm{H}), 0.90(\mathrm{~d}, J=6.6 \mathrm{~Hz}$, $6 \mathrm{H}), 0.79(\mathrm{t}, J=7.4 \mathrm{~Hz}, 3 \mathrm{H}) ;{ }^{13} \mathrm{C}$ NMR (126 MHz, DMSO-d6): $\delta$ (delta) ppm 176.5, 175.7, 102.1, 95.7, 85.1, 78.9, 77.8, 76.0, 75.4, 74.1, 73.6, 73.1, 70.8, 67.2, 65.4, 52.7, 50.6, 50.0, 49.4, 46.2, 45.1, 40.5, 40.0, 36.0, 35.4, 34.2, 28.6, 27.5, 25.6, 22.3, 21.7, 20.7, 16.0, 11.7; ${ }^{31} \mathrm{P}$ NMR (162 MHz, DMSO-d6): $\delta$ (delta) ppm -144.20 (sep) +24.04 (s); LC/MS $m / z=1178[\mathrm{M}]^{+}$; $\mathrm{RT}=2.22 \mathrm{~min}\left(\right.$ Method A); HRMS $\mathrm{m} / z[\mathrm{M}]^{+}$calcd for $\mathrm{C}_{67} \mathrm{H}_{106} \mathrm{~N}_{2} \mathrm{O}_{13} \mathrm{P}^{+}$1177.7427, found 1177.7378 .

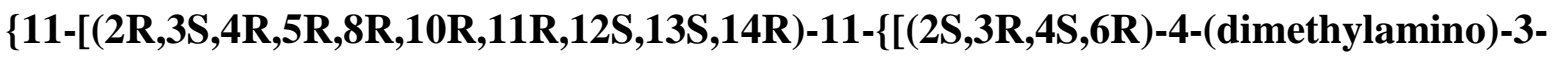
hydroxy-6-methyloxan-2-yl]oxy $\}$-2-ethyl-3,4,10-trihydroxy-13-\{[(2R,4R,5S,6S)-5hydroxy-4-methoxy-4,6-dimethyloxan-2-yl]oxy\}-3,5,8,10,12,14-hexamethyl-15-oxo-1oxa-6-azacyclopentadecan-6-yl]-11-oxoundecyl\}triphenylphosphonium chloride (2) 


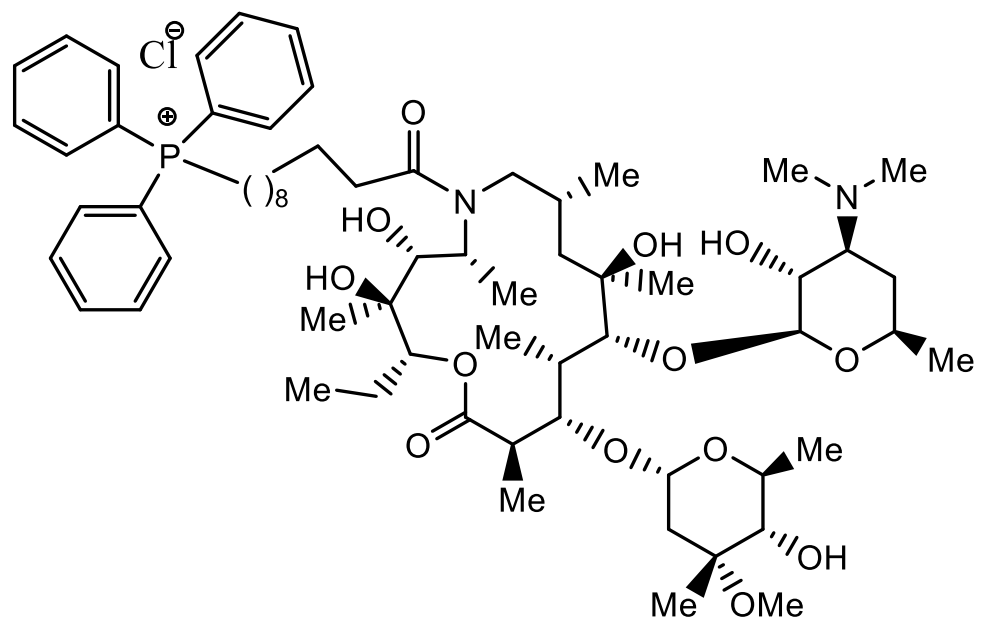

A stirred solution of (10-carboxydecyl)triphenylphosphonium bromide (431 mg, $0.82 \mathrm{mmol}$ ), 1-hydroxy-7-azabenzotriazole (HOAt) $(111 \mathrm{mg}, 0.82 \mathrm{mmol}), \mathrm{N}, \mathrm{N}$ '-diisopropylethylamine (DIEA) (0.36 mL, $2.04 \mathrm{mmol})$ and N,N'-diisopropylcarbodiimide (DIC) (127 $\mu \mathrm{L}, 0.82 \mathrm{mmol})$ in DCM $(2 \mathrm{~mL})$ was heated at $40{ }^{\circ} \mathrm{C}$. After $30 \mathrm{~min}$ the reaction mixture was added to a stirred solution of 9a-N-desmethyl-azithromycin $(0.5 \mathrm{~g} .0 .68 \mathrm{mmol})$ in DCM $(2 \mathrm{~mL})$ at $40{ }^{\circ} \mathrm{C}$. Stirring at $40{ }^{\circ} \mathrm{C}$ was continued overnight. On cooling to room temperature $\mathrm{H}_{2} \mathrm{O}(5 \mathrm{~mL})$ and DCM (5 $\mathrm{mL}$ ) were added and the mixture passed through a phase separator, washing with DCM $(2 \times 1$ $\mathrm{mL})$. The DCM fractions were pooled and solvent removed under reduced pressure to give a residue, which was taken up in $\mathrm{MeOH}(5 \mathrm{~mL})$ and heated at $60{ }^{\circ} \mathrm{C}$ for $2 \mathrm{~h}$. On cooling the solvent was removed under reduced pressure and the resulting residue taken up in THF (10 $\mathrm{mL})$, which was washed with saturated aqueous $\mathrm{NH}_{4} \mathrm{Cl}(10 \mathrm{~mL})$. The separated aqueous phase was further extracted with THF $(2 \times 5 \mathrm{~mL})$. The combined organic extracts were washed with brine $(10 \mathrm{~mL})$, dried over $\mathrm{MgSO}_{4}$ and solvent removed under reduced pressure. The resulting residue was purified by silica column chromatography eluting with $0-10 \% \mathrm{MeOH} / 0.7 \mathrm{M} \mathrm{NH}_{3}$ in DCM. The resulting product was partitioned between DCM $(10 \mathrm{~mL})$ and saturated aqueous $\mathrm{NH}_{4} \mathrm{Cl}(10 \mathrm{~mL})$. The separated organic phase was passed through a phase separator containing brine $(5 \mathrm{~mL})$. The collected solvent was evaporated under reduced pressure and the resulting residue taken up in $\mathrm{MeOH}(3 \mathrm{~mL})$ and filtered through an Amberlite IRA-400(Cl) ion exchange resin. The collected $\mathrm{MeOH}$ was recycled through the column $3 \mathrm{x}$, followed by a fresh volume of $\mathrm{MeOH}$. The combined $\mathrm{MeOH}$ washings were concentrated under reduced pressure to give the title compound 2 (400 mg, 49\% yield) as a white solid. ${ }^{1} \mathrm{H}$ NMR (400 MHz, DMSO- $d_{6}$ ): $\delta$ (delta) ppm 7.93-7.87 (m, 3H), 7.83-7.73 (m, 12H), $5.07(\mathrm{dd}, J=10.0,2.6 \mathrm{~Hz}, 1 \mathrm{H}), 4.75(\mathrm{~d}, J$ $=4.9 \mathrm{~Hz}, 1 \mathrm{H}), 4.72-4.54(\mathrm{~m}, 2 \mathrm{H}), 4.51-4.20(\mathrm{~m}, 3 \mathrm{H}), 4.16-3.99(\mathrm{~m}, 2 \mathrm{H}), 3.92(\mathrm{~d}, J=8.9 \mathrm{~Hz}$, $1 \mathrm{H}), 3.68-3.60(\mathrm{~m}, 1 \mathrm{H}), 3.60-3.51(\mathrm{~m}, 2 \mathrm{H}), 3.39(\mathrm{~d}, J=6.7 \mathrm{~Hz}, 1 \mathrm{H}), 3.29-3.23(\mathrm{~m}, 1 \mathrm{H}), 3.19$ $(\mathrm{s}, 3 \mathrm{H}), 3.04-2.94(\mathrm{~m}, 2 \mathrm{H}), 2.91(\mathrm{~d}, J=9.3 \mathrm{~Hz}, 1 \mathrm{H}), 2.71-2.61(\mathrm{~m}, 1 \mathrm{H}), 2.43-2.36(\mathrm{~m}, 1 \mathrm{H})$, 2.28-2.08 (m, 9H), 1.81-1.71 (m, 1H), 1.71-0.97 (m, 47H), 0.96-0.83 (m, 6H), 0.78 (t, $J=7.4$ $\mathrm{Hz}, 3 \mathrm{H}) ;{ }^{31} \mathrm{P}$ NMR $\left(162 \mathrm{MHz}, \mathrm{DMSO}-d_{6}\right): \delta$ (delta) ppm +24.06 (s); LC/MS $m / z=1164[\mathrm{M}]^{+}$; $\mathrm{RT}=1.93 \mathrm{~min}\left(\right.$ Method A); HRMS $\mathrm{m} / z[\mathrm{M}]^{+}$calcd for $\mathrm{C}_{66} \mathrm{H}_{104} \mathrm{~N}_{2} \mathrm{O}_{13} \mathrm{P}^{+}$1163.7270, found 1163.7229 .

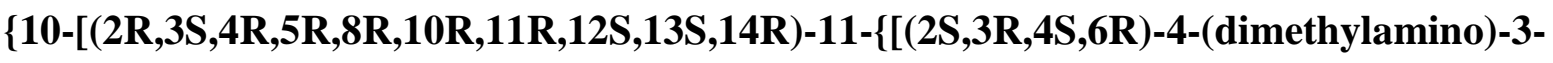
hydroxy-6-methyloxan-2-yl]oxy $\}$-2-ethyl-3,4,10-trihydroxy-13- $\{[(2 \mathrm{R}, 4 \mathrm{R}, 5 \mathrm{~S}, 6 \mathrm{~S})-5$ hydroxy-4-methoxy-4,6-dimethyloxan-2-yl]oxy\}-3,5,8,10,12,14-hexamethyl-15-oxo-1- 
oxa-6-azacyclopentadecan-6-yl]-10-oxodecyl\}triphenylphosphonium hexafluorophosphate(V) (3)

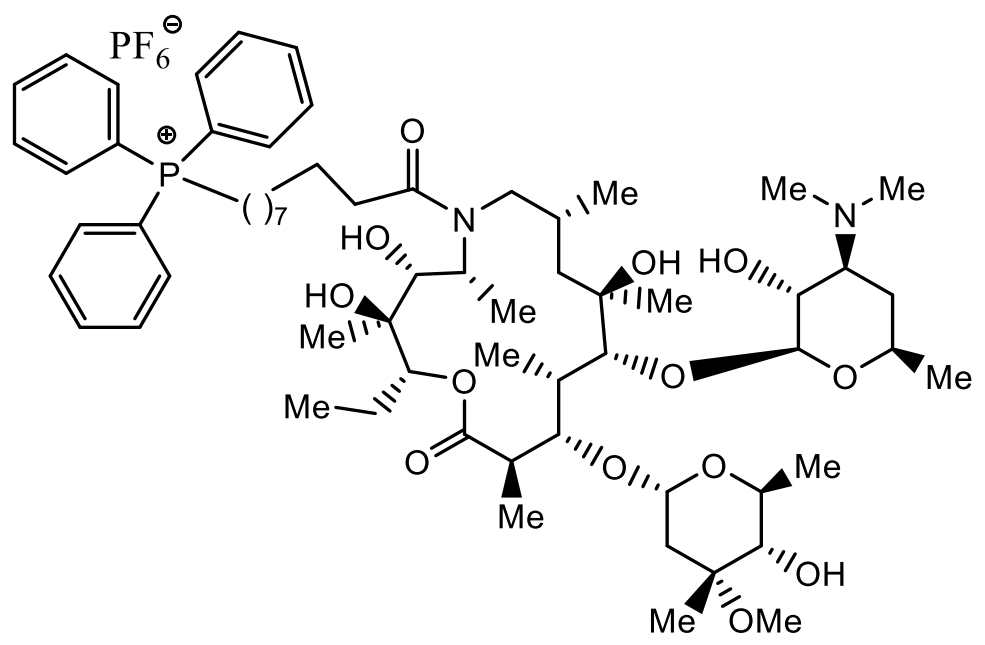

Prepared as described for (1) using (9-carboxynonyl)triphenylphosphonium bromide as a white solid. ${ }^{1} \mathrm{H}$ NMR (400 MHz, DMSO- $\left.d_{6}\right): \delta$ (delta) ppm 7.93-7.85 (m, 3H), 7.84-7.72 (m, 13H), $5.08(\mathrm{dd}, J=10.1,2.5 \mathrm{~Hz}, 1 \mathrm{H}), 4.88-4.78(\mathrm{~m}, 1 \mathrm{H}), 4.75(\mathrm{~d}, J=4.8 \mathrm{~Hz}, 1 \mathrm{H}), 4.72-4.48(\mathrm{~m}, 2 \mathrm{H})$, $4.40(\mathrm{~d}, 1 \mathrm{H}), 4.37-4.26(\mathrm{~m}, 1 \mathrm{H}), 4.15-3.98(\mathrm{~m}, 2 \mathrm{H}), 3.92(\mathrm{~d}, J=9.0 \mathrm{~Hz}, 1 \mathrm{H}), 3.70-3.60(\mathrm{~m}$, $1 \mathrm{H}), 3.60-3.50(\mathrm{~m}, 3 \mathrm{H}), 3.23-3.15(\mathrm{~m}, 5 \mathrm{H}), 3.05-2.86(\mathrm{~m}, 3 \mathrm{H}), 2.71-2.56(\mathrm{~m}, 1 \mathrm{H}), 2.44-2.35$ (m, 1H), 2.28-2.09 (m, 9H), 1.84-1.71 (m, 1H), 1.63-1.38 (m, 9H), 1.37-0.93 (m, 33H), 0.89 $(\mathrm{d}, J=6.3 \mathrm{~Hz}, 4 \mathrm{H}), 0.78(\mathrm{t}, J=7.5 \mathrm{~Hz}, 6 \mathrm{H}) ;{ }^{31} \mathrm{P}$ NMR $\left(162 \mathrm{MHz}, \mathrm{DMSO}-d_{6}\right): \delta$ (delta) ppm $144.13(\mathrm{sep}),+24.06(\mathrm{~s}) ; \mathrm{LC} / \mathrm{MS} \mathrm{m} / z=1150[\mathrm{M}]^{+} ; \mathrm{RT}=1.94 \min (\operatorname{Method} \mathrm{A})$.

\{9-[(2R,3S,4R,5R,8R,10R,11R,12S,13S,14R)-11-\{[(2S,3R,4S,6R)-4-(dimethylamino)-3hydroxy-6-methyloxan-2-yl]oxy $\}$-2-ethyl-3,4,10-trihydroxy-13-\{[(2R,4R,5S,6S)-5hydroxy-4-methoxy-4,6-dimethyloxan-2-yl]oxy\}-3,5,8,10,12,14-hexamethyl-15-oxo-1oxa-6-azacyclopentadecan-6-yl]-9-oxononyl\}triphenylphosphonium hexafluorophosphate(V) (4)

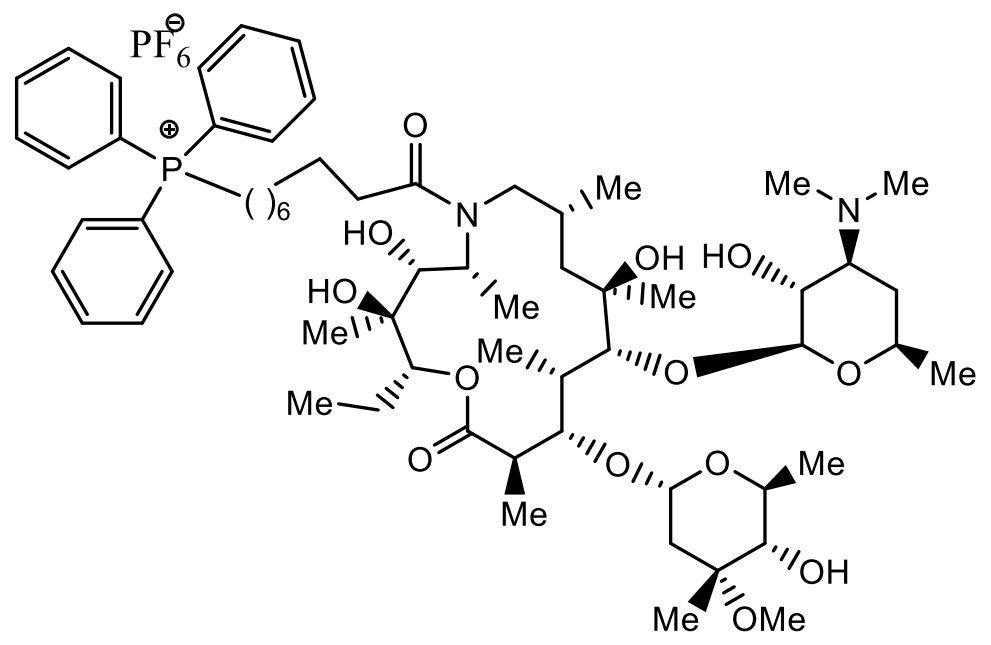

Prepared as described for (1) using (8-carboxyoctyl)triphenylphosphonium as a white solid. ${ }^{1} \mathrm{H}$ NMR (400 MHz, DMSO- $\left.d_{6}\right): \delta$ (delta) ppm 7.94-7.86 (m, 3H), 7.84-7.73 (m, 12H), 5.06 (d, $J$ $=10.0 \mathrm{~Hz}, 1 \mathrm{H}), 4.93-4.86(\mathrm{~m}, 1 \mathrm{H}), 4.75(\mathrm{~d}, J=5.0 \mathrm{~Hz}, 2 \mathrm{H}), 4.42(\mathrm{q}, J=5.8,4.2 \mathrm{~Hz}, 1 \mathrm{H}), 4.31$ $(\mathrm{d}, J=7.6 \mathrm{~Hz}, 1 \mathrm{H}), 4.25(\mathrm{~d}, J=7.6 \mathrm{~Hz}, 1 \mathrm{H}), 4.12-3.99(\mathrm{~m}, 2 \mathrm{H}), 3.94(\mathrm{~d}, J=8.7 \mathrm{~Hz}, 1 \mathrm{H}), 3.66$ 
$(\mathrm{s}, 1 \mathrm{H}), 3.53(\mathrm{~d}, J=15.4 \mathrm{~Hz}, 2 \mathrm{H}), 3.48-3.35(\mathrm{~m}, 2 \mathrm{H}), 3.21(\mathrm{~d}, J=18.9 \mathrm{~Hz}, 3 \mathrm{H}), 3.06-2.87(\mathrm{~m}$, $3 \mathrm{H}), 2.78-2.54(\mathrm{~m}, 3 \mathrm{H}), 2.41(\mathrm{~d}, J=16.8 \mathrm{~Hz}, 2 \mathrm{H}), 2.24(\mathrm{~d}, J=13.4 \mathrm{~Hz}, 7 \mathrm{H}), 1.76(\mathrm{~s}, 3 \mathrm{H}), 1.55-$ $1.33(\mathrm{~m}, 9 \mathrm{H}), 1.32-1.05(\mathrm{~m}, 30 \mathrm{H}), 1.04-0.93(\mathrm{~m}, 3 \mathrm{H}), 0.89(\mathrm{~d}, J=6.4 \mathrm{~Hz}, 4 \mathrm{H}), 0.80(\mathrm{dt}, J=$ 15.0, $5.4 \mathrm{~Hz}, 3 \mathrm{H}) ;{ }^{31} \mathrm{P}$ NMR (162 MHz, DMSO- $d_{6}$ ): $\delta$ (delta) ppm -146.39 (sep), +24.02 (s); $\mathrm{LC} / \mathrm{MS} m / z=978[\mathrm{M} \text {-cladinsoyl }]^{+} ; \mathrm{RT}=1.99 \min ($ Method A $)$.

\{8-[(2R,3S,4R,5R,8R,10R,11R,12S,13S,14R)-11-\{[(2S,3R,4S,6R)-4-(dimethylamino)-3hydroxy-6-methyloxan-2-yl]oxy\}-2-ethyl-3,4,10-trihydroxy-13-\{[(2R,4R,5S,6S)-5hydroxy-4-methoxy-4,6-dimethyloxan-2-yl]oxy\}-3,5,8,10,12,14-hexamethyl-15-oxo-1oxa-6-azacyclopentadecan-6-yl]-8-oxooctyl\}triphenylphosphonium hexafluorophosphate(V) (5)

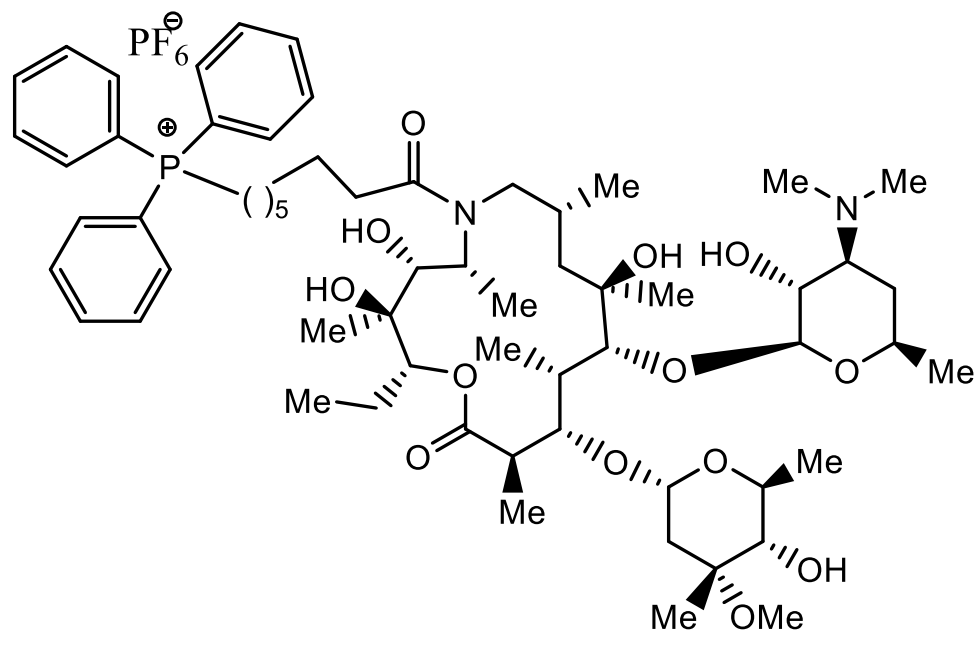

Prepared as described for (1) using (7-carboxyheptyl)triphenylphosphonium bromide as a white solid. ${ }^{1} \mathrm{H}$ NMR (400 MHz, DMSO- $\left.d_{6}\right)$ : $\delta$ (delta) ppm 7.93-7.86 (m, 3H), 7.84-7.73 (m, $12 \mathrm{H}), 4.94-4.85(\mathrm{~m}, 1 \mathrm{H}), 4.71-4.57(\mathrm{~m}, 1 \mathrm{H}), 4.53-4.35(\mathrm{~m}, 2 \mathrm{H}), 4.09-4.00(\mathrm{~m}, 1 \mathrm{H}), 4.00-3.93$ $(\mathrm{m}, 1 \mathrm{H}), 3.79-3.65(\mathrm{~m}, 1 \mathrm{H}), 3.64-3.51(\mathrm{~m}, 3 \mathrm{H}), 3.23(\mathrm{~s}, 3 \mathrm{H}), 3.17-3.00(\mathrm{~m}, 2 \mathrm{H}), 2.98-2.85(\mathrm{~m}$, $2 \mathrm{H}), 2.75-2.53(\mathrm{~m}, 2 \mathrm{H}), 2.40-2.12(\mathrm{~m}, 4 \mathrm{H}), 2.09(\mathrm{~s}, 5 \mathrm{H}), 2.05-1.73(\mathrm{~m}, 4 \mathrm{H}), 1.71-1.62(\mathrm{~m}, 1 \mathrm{H})$, 1.60-1.32 (m, 9H), 1.30-1.05 (m, 27H), 1.03-0.89 (m, 7H), 0.88-0.68 (m, 7H); ${ }^{31} \mathrm{P}$ NMR (162 MHz, DMSO- $d_{6}$ ): $\delta$ (delta) ppm -144.22 (sep), +24.01 (s); LC/MS m/z=1122 [M] ${ }^{+}$; RT = 1.59 min (Method A).

(11-\{[(5R,5aR,6S,6aR,7S,10aS)-9-carbamoyl-7-(dimethylamino)-1,6,8,10a,11pentahydroxy-5-methyl-10,12-dioxo-5,5a,6,6a,7,10,10a,12-octahydrotetracen-2yl]carbamoyl\}undecyl)triphenylphosphonium chloride $\mathrm{HCl}$ (6)<smiles>C[C@H]1c2ccc(NC(=O)CCO)c(O)c2C(=O)C2=C(O)[C@]3(O)C(=O)C(C(N)=O)=C(O)[C@H](N(C)C)[C@]3(C)[C@@H](O)[C@]21C</smiles>

To a solution of (11-carboxyundecyl)triphenylphosphonium bromide (1.20 g, $2.22 \mathrm{mmol})$ in DCM (5 mL) was added 1-chloro-N,N-2-trimethyl-1-propenylamine (Ghosez's reagent) (400 
$\mu \mathrm{L}, 3.02 \mathrm{mmol}$ ). The resulting mixture was stirred at room temperature for $10 \mathrm{~min}$ and then added dropwise to a stirred solution of 9-aminodoxycyline dihydrochloride $(1 \mathrm{~g}, 2.02 \mathrm{mmol})$ in DMF $(6 \mathrm{~mL})$ at $0{ }^{\circ} \mathrm{C}$. The resulting reaction mixture was warmed to room temperature and treated with concentrated $\mathrm{HCl}(200 \mu \mathrm{L}, 2.60 \mathrm{mmol})$. The reaction mixture was diluted with DCM $(20 \mathrm{~mL})$ and added dropwise to stirring cold MTBE $(500 \mathrm{~mL})$. The resulting precipitate was collected by filtration, washed with fresh MTBE and dried under vacuo. The resulting crude product was purified by preparative HPLC (Waters X-Select Prep-C18 $5 \mu \mathrm{m}, 19$ x 50 mm eluting with $\left.\mathrm{MeCN} / \mathrm{H}_{2} \mathrm{O} / 0.1 \% \mathrm{HCOOH}\right)$ to give a solid, which was taken up in $\mathrm{MeOH}(3$ $\mathrm{mL})$ and diluted with DCM $(20 \mathrm{~mL})$. The resulting solution was added slowly to stirring $\mathrm{Et}_{2} \mathrm{O}$ and the precipitate collected by filtration, taken up into $\mathrm{MeOH}(2 \mathrm{~mL})$ and filtered through an Amberlite IRA-400(Cl) ion exchange resin. The collected $\mathrm{MeOH}$ was recycled through the column, followed by a fresh volume of $\mathrm{MeOH}$. The combined $\mathrm{MeOH}$ washings were concentrated under reduced pressure to give the title compound 6 (607 $\mathrm{mg}, 31 \%$ yield) as a dark yellow solid. ${ }^{1} \mathrm{H}$ NMR (500 MHz, DMSO- $d_{6}$ ): $\delta$ (delta) ppm 15.01 (s, 1H), $11.98(\mathrm{~s}, 1 \mathrm{H})$, 10.24 (br. s, 1H), $9.56(\mathrm{~s}, 1 \mathrm{H}), 9.29(\mathrm{~s}, 1 \mathrm{H}), 9.02(\mathrm{~s}, 1 \mathrm{H}), 8.09$ (d, $J=8.3 \mathrm{~Hz}, 1 \mathrm{H}), 7.89$ (tt, $J=$ 7.0, 1.9 Hz, 3H), 7.84-7.74 (m, 12H), 7.55 (br. s, 1H), 6.90 (d, J = 8.3 Hz, 1H), $6.22(\mathrm{~s}, 1 \mathrm{H})$, $4.75(\mathrm{~s}, 1 \mathrm{H}), 3.62-3.52(\mathrm{~m}, 2 \mathrm{H}), 3.52-3.45(\mathrm{~m}, 1 \mathrm{H}), 2.90(\mathrm{~d}, J=11.3 \mathrm{~Hz}, 1 \mathrm{H}), 2.81$ (br. s, 6H), 2.67-2.59 (m, 1H), 2.59-2.51 (m, 1H), 2.42-2.36 (m, 2H), 1.61-1.40 (m, 7H), 1.33-1.14 (m, 14H). ${ }^{31} \mathrm{P}$ NMR (202 MHz, DMSO- $\left.d_{6}\right): \delta$ (delta) ppm +24.08 (s); LC/MS $m / z=903[\mathrm{M}]^{+} ; \mathrm{RT}$ $=3.35 \mathrm{~min}($ Method $\mathrm{B})$.

(10-\{[(5R,5aR,6S,6aR,7S,10aS)-9-carbamoyl-7-(dimethylamino)-1,6,8,10a,11pentahydroxy-5-methyl-10,12-dioxo-5,5a,6,6a,7,10,10a,12-octahydrotetracen-2yl]carbamoyl\}decyl)triphenylphosphonium chloride $\mathrm{HCl}$ (7)<smiles>C[C@H]1c2ccc(NC(=O)CC[Te][PH](c3ccccc3)(c3ccccc3)c3ccccc3)c(O)c2C(=O)C2=C(O)[C@@]3(O)C(=O)C(C(N)=O)=C(O)[C@H](N(C)C)[C@@H]3[C@@H](O)[C@]21C</smiles>

Prepared as described for (6) using (9-carboxynonyl)triphenylphosphonium bromide. After preparative HPLC the resulting brown solid was taken up in $\mathrm{MeOH}(2 \mathrm{~mL})$ and filtered through an Amberlite IRA-400(Cl) ion exchange resin. The collected $\mathrm{MeOH}$ was recycled through the column, followed by a fresh volume of $\mathrm{MeOH}$. The combined $\mathrm{MeOH}$ washings were concentrated under reduced pressure to give the title compound 7 as a yellow brown solid. ${ }^{1} \mathrm{H}$ NMR (500 MHz, DMSO-d6): $\delta$ (delta) ppm 15.01 (s, 1H), 11.96 (s, 1H), 10.11 (s, 1H), 9.55 (s, $1 \mathrm{H}), 9.28(\mathrm{~s}, 1 \mathrm{H}), 9.04(\mathrm{~s}, 1 \mathrm{H}), 8.09(\mathrm{~d}, J=8.3 \mathrm{~Hz}, 1 \mathrm{H}), 7.93-7.86(\mathrm{~m}, 3 \mathrm{H}), 7.83-7.72(\mathrm{~m}, 12 \mathrm{H})$, $7.58(\mathrm{~s}, 1 \mathrm{H}), 6.91(\mathrm{~d}, J=8.4 \mathrm{~Hz}, 1 \mathrm{H}), 5.94(\mathrm{~s}, 1 \mathrm{H}), 4.55(\mathrm{~s}, 1 \mathrm{H}), 3.60-3.49(\mathrm{~m}, 3 \mathrm{H}), 2.89-2.72$ $(\mathrm{m}, 7 \mathrm{H}), 2.69-2.60(\mathrm{~m}, 1 \mathrm{H}), 2.57-2.52(\mathrm{~m}, 1 \mathrm{H}), 2.42-2.35(\mathrm{~m}, 2 \mathrm{H}), 1.61-1.38(\mathrm{~m}, 9 \mathrm{H}), 1.32-$ $1.16(\mathrm{~m}, 10 \mathrm{H}) ;{ }^{31} \mathrm{P}$ NMR $\left(202 \mathrm{MHz}, \mathrm{DMSO}-d_{6}\right): \delta$ (delta) ppm $+24.05(\mathrm{~s}) ; \mathrm{LC} / \mathrm{MS} \mathrm{m} / z=888.5$ $[\mathrm{M}]^{+} ; \mathrm{RT}=4.26 \mathrm{~min}($ Method B).

(9-\{[(5R,5aR,6S,6aR,7S,10aS)-9-carbamoyl-7-(dimethylamino)-1,6,8,10a,11pentahydroxy-5-methyl-10,12-dioxo-5,5a,6,6a,7,10,10a,12-octahydrotetracen-2yl]carbamoyl\}nonyl)triphenylphosphonium chloride $\mathrm{HCl}(8)$ 
<smiles></smiles>

Prepared as described for (7) using (9-carboxynonyl)triphenylphosphonium bromide as a yellow solid. ${ }^{1} \mathrm{H}$ NMR (500 MHz, DMSO- $d_{6}$ ): $\delta$ (delta) ppm 15.01 (brs, $\left.1 \mathrm{H}\right), 12.02(\mathrm{~s}, 1 \mathrm{H})$, $9.28(\mathrm{~s}+\mathrm{m}, 2 \mathrm{H}), 9.10-8.90(\mathrm{~m}, 1 \mathrm{H}), 8.09(\mathrm{~d}, J=8.3 \mathrm{~Hz}, 1 \mathrm{H}), 7.92-7.86(\mathrm{~m}, 3 \mathrm{H}), 7.84-7.72(\mathrm{~m}$, $13 \mathrm{H}), 6.90(\mathrm{~d}, J=8.4 \mathrm{~Hz}, 1 \mathrm{H}), 5.93(\mathrm{bs}, 1 \mathrm{H}), 4.44$ (brs, $1 \mathrm{H}), 3.63-3.50(\mathrm{~m}, 3 \mathrm{H}), 2.85-2.62(\mathrm{~m}$, $8 \mathrm{H}), 2.54(\mathrm{dd}, J=12.4,7.8 \mathrm{~Hz}, 1 \mathrm{H}), 2.39$ (t, $J=7.4 \mathrm{~Hz}, 2 \mathrm{H}), 1.61-1.49(\mathrm{~m}, 4 \mathrm{H}), 1.48-1.37$ (m, $6 \mathrm{H}), 1.35-1.11(\mathrm{~m}, 11 \mathrm{H}) ;{ }^{31} \mathrm{P}$ NMR $\left(202 \mathrm{MHz}, \mathrm{DMSO}-d_{6}\right): \delta$ (delta) ppm $+24.08(\mathrm{~s})$; LC/MS $m / z=874.7[\mathrm{M}]^{+} ; \mathrm{RT}=3.94 \min ($ Method $\mathrm{B})$.

(8-(\{[(5R,5aR,6S,6aR,7S,10aS)-9-carbamoyl-7-(dimethylamino)-1,6,8,10a,11pentahydroxy-5-methyl-10,12-dioxo-5,5a,6,6a,7,10,10a,12-octahydrotetracen-2yl]carbamoyl\}octyl)phosphonium chloride $\mathrm{HCl}$ (9)<smiles>C[C@H]1c2ccc(NC(=O)CCO)c(O)c2C(=O)C2=C(O)[C@]3(O)C(=O)C(C(N)=O)=C(O)[C@H](N(C)C)[C@@H]3[C@@H](O)[C@]21C</smiles>

Prepared as described for (7) using (8-carboxyoctyl)triphenylphosphonium bromide as a yellow solid. ${ }^{1} \mathrm{H}$ NMR (500 MHz, DMSO- $d_{6}$ ): $\delta$ (delta) ppm $15.01(\mathrm{~s}, 1 \mathrm{H}), 11.96(\mathrm{~s}, 1 \mathrm{H}), 10.07$ $(\mathrm{s}, 1 \mathrm{H}), 9.54(\mathrm{~s}, 1 \mathrm{H}), 9.27(\mathrm{~s}, 1 \mathrm{H}), 9.05(\mathrm{~s}, 1 \mathrm{H}), 8.09(\mathrm{~d}, J=8.3 \mathrm{~Hz}, 1 \mathrm{H}), 7.92-7.87(\mathrm{~m}, 3 \mathrm{H})$, 7.83-7.73 (m, 12H), 7.59 (s, 1H), $6.91(\mathrm{dd}, J=8.5,1.1 \mathrm{~Hz}, 1 \mathrm{H}), 5.86(\mathrm{~s}, 1 \mathrm{H}), 4.49(\mathrm{~s}, 1 \mathrm{H})$, 3.60-3.50 (m, 3H), 2.93-2.70 (m, 7H), 2.69-2.61 (m, 1H), $2.53(\mathrm{dd}, J=12.0,7.9 \mathrm{~Hz}, 1 \mathrm{H}), 2.41-$ 2.33 (m, 2H), 1.60-1.49 (m, 4H), 1.48-1.38 (m, 5H), 1.33-1.18 (m, 6H); ${ }^{31} \mathrm{P}$ NMR $(202 \mathrm{MHz}$, DMSO- $\left.d_{6}\right): \delta$ (delta) ppm +24.06; LC/MS $\mathrm{m} / z=861[\mathrm{M}]^{+} ; \mathrm{RT}=3.68 \min ($ Method B).

(7-\{[(5R,5aR,6S,6aR,7S,10aS)-9-carbamoyl-7-(dimethylamino)-1,6,8,10a,11pentahydroxy-5-methyl-10,12-dioxo-5,5a,6,6a,7,10,10a,12-octahydrotetracen-2yl]carbamoyl\}heptyl)triphenylphosphonium trifluoroacetate $\mathrm{CF}_{3} \mathrm{COOH}$ (10)

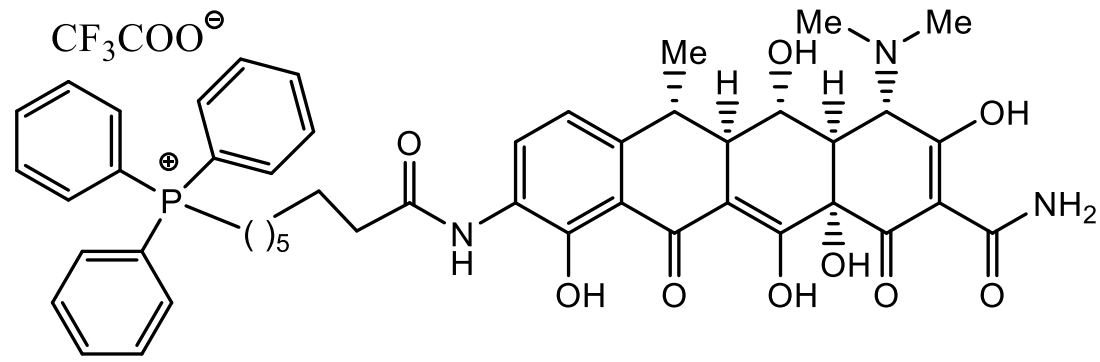


To (7-carboxyheptyl)triphenylphosphonium bromide $(53 \mathrm{mg}, 0.13 \mathrm{mmol})$ in DCM $(3.5 \mathrm{~mL})$ over $4 \AA$ molecular sieves was added N,N'-diisopropylethylamine (DIEA) (63 $\mu \mathrm{L}, 0.36 \mathrm{mmol}$ ) followed by propylphosphonic anhydride (T3P) $50 \%$ wt in DCM (93 mg, $0.15 \mathrm{mmol})$. To the reaction mixture was added 9-aminodoxycyline dihydrochloride $(80 \mathrm{mg}, 0.15 \mathrm{mmol})$. After stirring at room temperature for $16 \mathrm{~h}$ the reaction mixture was filtered through celite, which was washed with DCM. The combined organics were evaporated under reduced pressure and the resulting residue purified by preparative HPLC (Gemini NX-C18 $10 \mu \mathrm{m}, 30$ x $250 \mathrm{~mm}$ eluting with a mixture of $\mathrm{MeCN} / 0.035 \% \mathrm{CF}_{3} \mathrm{COOH}$ and $\mathrm{H}_{2} \mathrm{O} / 0.05 \% \mathrm{CF}_{3} \mathrm{COOH}$ ) to give the title compound 10 (28 mg, $18 \%$ yield) as a yellow solid. ${ }^{1} \mathrm{H} \mathrm{NMR}\left(400 \mathrm{MHz}, \mathrm{CD}_{3} \mathrm{OD}\right): \delta$ (delta) ppm $8.16(\mathrm{~d}, J=8.4 \mathrm{~Hz}, 1 \mathrm{H}), 7.94-7.86(\mathrm{~m}, 3 \mathrm{H}), 7.86-7.73(\mathrm{~m}, 12 \mathrm{H}), 6.97(\mathrm{~d}, J=8.4 \mathrm{~Hz}, 1 \mathrm{H})$, $4.45(\mathrm{~s}, 1 \mathrm{H}), 3.59$ (dd, $J=11.5,8.3 \mathrm{~Hz}, 1 \mathrm{H}), 3.46-3.37(\mathrm{~m}, 2 \mathrm{H}), 2.98(\mathrm{~d}, J=27.7 \mathrm{~Hz}, 6 \mathrm{H}), 2.86$ $(\mathrm{dd}, J=11.4,1.1 \mathrm{~Hz}, 1 \mathrm{H}), 2.83-2.72(\mathrm{~m}, 1 \mathrm{H}), 2.61(\mathrm{dd}, J=12.3,8.3 \mathrm{~Hz}, 1 \mathrm{H}), 2.46(\mathrm{t}, J=7.3$ $\mathrm{Hz}, 2 \mathrm{H}), 1.77-1.65(\mathrm{~m}, 4 \mathrm{H}), 1.65-1.54(\mathrm{~m}, 5 \mathrm{H}), 1.49-1.34(\mathrm{~m}, 4 \mathrm{H}) ;{ }^{31} \mathrm{P}$ NMR $(162 \mathrm{MHz}$, $\left.\mathrm{CD}_{3} \mathrm{OD}\right): \delta$ (delta) ppm +23.79 (s); LC/MS m/z=846.6 [M] ${ }^{+} ; \mathrm{RT}=2.44 \mathrm{~min}($ Method B); HRMS $m / z[\mathrm{M}]^{+}$calcd for $\mathrm{C}_{48} \mathrm{H}_{53} \mathrm{~N}_{3} \mathrm{O}_{9} \mathrm{P}^{+} 846.3514$, found 846.3474.

\{12-[(2R,3S,4R,5R,8R,10R,11R,12S,13S,14R)-2-ethyl-3,4,10,11,13-pentahydroxy3,5,8,10,12,14-hexamethyl-15-oxo-1-oxa-6-azacyclopentadecan-6-yl]-12oxododecyl\}triphenylphosphonium hexafluorophosphate(V) (18)

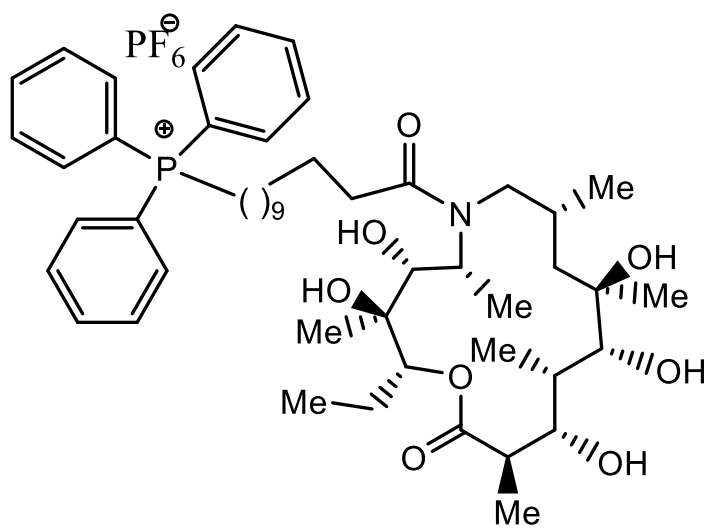

Prepared as described for (1) using (2R,3S,4R,5R,8R,10R,11R,12S,13S, 14R)-2-ethyl3,4,10,11,13-pentahydroxy-3,5,8,10,12,14-hexamethyl-15-oxo-1-oxa-6-azacyclopentadecan15-one (prepared as described in WO201111636312) as a white solid. ${ }^{1} \mathrm{H}$ NMR (500 MHz, DMSO- $\left.d_{6}\right): \delta$ (delta) ppm 7.94-7.87 (m, 3H), 7.85-7.74 (m, 12H), 5.19-5.07 (m, 1H), 4.75-4.59 $(\mathrm{m}, 2 \mathrm{H}), 4.59-4.46(\mathrm{~m}, 1 \mathrm{H}), 4.36-4.20(\mathrm{~m}, 2 \mathrm{H}), 3.63-3.51(\mathrm{~m}, 2 \mathrm{H}), 3.45-3.34(\mathrm{~m}, 1 \mathrm{H}), 3.31-$ $3.17(\mathrm{~m}, 2 \mathrm{H}), 3.06-2.70(\mathrm{~m}, 2 \mathrm{H}), 2.44-2.27(\mathrm{~m}, 2 \mathrm{H}), 2.20-2.00(\mathrm{~m}, 2 \mathrm{H}), 1.87-1.73(\mathrm{~m}, 1 \mathrm{H})$, $1.59-1.40(\mathrm{~m}, 8 \mathrm{H}), 1.39-1.32(\mathrm{~m}, 1 \mathrm{H}), 1.32-1.14(\mathrm{~m}, 21 \mathrm{H}), 1.08(\mathrm{dd}, J=16.0,6.8 \mathrm{~Hz}, 4 \mathrm{H})$, 0.95-0.84 (m, 4H), 0.79 (t, $J=7.5 \mathrm{~Hz}, 3 \mathrm{H}), 0.72-0.59$ (m, 3H); ${ }^{31} \mathrm{P}$ NMR (202 MHz, DMSO$\left.d_{6}\right): \delta$ (delta) ppm -144.15 (sep), $+24.08\left(\mathrm{~d}, J=1.8 \mathrm{~Hz}\right.$ ); LC/MS $m / z=863[\mathrm{M}]^{+} ; \mathrm{RT}=1.65$ $\min ($ Method A).

\section{Mitochondrial translation inhibition}

MDA-MB-231 (ATCC@ HTB-26) green labelled cells were maintained in culture in DMEM with $10 \%$ of foetal bovine serum tetracycline-free (FBS tet-free, PAN Biotech P30-3602). They were seeded into 6-cm dishes at density of 150,000 cells/dish and transferred into a hypoxia chamber containing $3 \% \mathrm{O}_{2}\left(37{ }^{\circ} \mathrm{C}, 5 \% \mathrm{CO}_{2}\right)$. Cells were treated with test compound the next day and left for $72 \mathrm{~h}$ before collection in ice-cold RIPA buffer (Millipore, 20-188) containing 
protease inhibitor cocktail (Promega, G6521) and phosphatase inhibitor 2 and 3 (Sigma, P5726 and $\mathrm{P} 0044$ respectively). Lysate was transferred into a pre-chilled $1.5 \mathrm{ml}$ Eppendorf tube and sonicated for $10 \mathrm{sec}$. Lysate was then centrifuged at $13,000 \mathrm{rpm}$ for $5 \mathrm{~min}$ at $4{ }^{\circ} \mathrm{C}$. The resulting pellet was discarded, and supernatant total protein content was quantified using the $D C$ protein assay (BioRad, 500-0116). Samples (10 $\mu \mathrm{g}$ total protein) were separated with SDS-PAGE (BioRad) and blotted for MTCO1 (Abcam, ab14705, 1/1000) and actin (Abcam, ab6276, 1/4000) using iBind Flex system according to manufacturer instructions (Invitrogen). Bands were imaged with Chemidoc (BioRad) and quantified using Image Lab software (BioRad). Data were normalised to actin then DMSO control.

\section{Mitochondrial Bioenergetics}

Oxygen consumption rates (OCR) was measured utilizing the Seahorse XFe96 Extracellular Flux Analyzer (Agilent Technology). MDA-MB-231 (ATCC® HTB-26) green labelled cells were treated with test compound $\left(37{ }^{\circ} \mathrm{C}, 5 \% \mathrm{CO}_{2}\right)$ for $72 \mathrm{~h}$ and then seeded $16 \mathrm{~h}$ before performing OCR analysis into a collagen 1 pre-coated 96-well Seahorse microplate at a density of 12,500 cells per well. Just prior the assay, cells were washed 3 times with assay medium (XF base medium [Agilent, 103334-100] containing $10 \mathrm{mM}$ D-glucose, $2 \mathrm{mM}$ L-glutamine and $1 \mathrm{mM}$ sodium pyruvate) containing $1.5 \%$ foetal bovine serum tetracycline-free (FBS tetfree, PAN Biotech P30-3602). Then the plate was incubated for $1 \mathrm{~h}$ in a non- $\mathrm{CO}_{2}$ incubator before measuring the OCR. Three baseline OCR measurement were recorded before injecting oligomycin A $(1 \mu \mu \mathrm{M})$ to inhibit ATP synthase, carbonyl cyanide-ptrifluoromethoxyphenylhydrazone (FCCP, $1.2 \mu \mathrm{M})$ to uncouple the mitochondria and yield maximal OCR, and rotenone $(0.5 \mu \mathrm{M})$ and antimycin $\mathrm{A}(0.5 \mu \mathrm{M})$ to prevent mitochondrial oxygen consumption through inhibition of Complex I and Complex III, respectively. From these measurements, indices of mitochondrial function were determined. Data were normalised to cell number.

\section{Cell viability}

MDA-MB-231 (ATCC® HTB-26) green labelled cells were seeded into a 96-well plate at a density of 2,500 cells per well and left to adhere overnight in a hypoxia chamber containing $3 \% \mathrm{O}_{2}\left(37^{\circ} \mathrm{C}, 5 \% \mathrm{CO}_{2}\right)$. Cells were then treated with test compound at various concentrations (up to $5 \mu \mathrm{M}$ ) and cell number were counted overtime using an Incucyte ZOOM imaging system (Essen Bioscience) in a hypoxic incubator. EC50 was generated using data from $108 \mathrm{~h}$ posttreatment and calculated with GraphPad PrismTM version 8.0 (GraphPad Software, Inc., San Diego, CA, USA). 